Conexões entre Homens e Saúde: discutindo algumas arranhaduras da masculinidade

\title{
Connections between Men and Health: discussing some scratches of masculinity
}

\author{
Andréia Burille, Tatiana Engel Gerhardt \\ Universidade Federal do Rio Grande do Sul, tatiana.gerhardt@ufrgs.br
}

\begin{tabular}{|c|c|}
\hline & Resumo \\
\hline Palabras clave & Neste artigo busca-se discutir as conexões entre homens e saúde abordando \\
\hline $\begin{array}{l}\text { Saúde } \\
\text { Homens } \\
\text { Masculinidade }\end{array}$ & $\begin{array}{l}\text { algumas arranhaduras da masculinidade. No primeiro momento, trazem-se alguns } \\
\text { dados oriundos do seminário temático "Ser homem na contemporaneidade: } \\
\text { discutindo algumas arranhaduras da masculinidade" apresentado no Seminário } \\
\text { Internacional Rotas Críticas III, realizado na cidade de Porto Alegre, Brasil, em } \\
\text { 2011. Por conseguinte, discute-se o processo de socialização masculino e pontua- } \\
\text { se alguns aspectos da vida cotidiana que podem colocar em risco/ameaçar ou } \\
\text { arranhar a masculinidade, como buscar cuidado e até mesmo o próprio processo } \\
\text { de envelhecimento. Assinala-se que a masculinidade heteronormativa configura-se } \\
\text { como modelo hegemônico, sobressaindo sobre os demais modelos. Assim, ser } \\
\text { homem é cumprir papéis e prescrições, que estão enraizadas em uma sociedade } \\
\text { que se estrutura em função do gênero, mesmo quando colocam em risco sua } \\
\text { saúde. }\end{array}$ \\
\hline
\end{tabular}

\section{Abstract}

\section{Keywords}

Health

Men

Masculinity

\begin{abstract}
In this article seeks to discuss the connections between men and health addressing some scratches of masculinity. At first, bring up some data from the thematic seminar "Being a man today: discussing some scratches masculinity," presented at the International Seminar Reviews Routes III, held in Porto Alegre, Brazil, in 2011. Therefore, we discuss the process of male socialization and scores some aspects of everyday life that can endanger / or scratch threaten masculinity, such as seeking care and even the aging process itself. It is noted that the heteronormative masculinity configures itself as the hegemonic model, stand out among other models. So, being a man is to fulfill roles and prescriptions which are rooted in a society that is structured on the basis of gender, even for this have to endanger your health.
\end{abstract}

\section{Introdução}

Conhecer as conexões entre homens e saúde é o desafio que tem nos motivado desde 2009. Por ser um tema recente ainda há lacunas quando se fala das relações estabelecidas entre ser homem, cuidado com a saúde e masculinidade, que tem nos impulsionado a seguir realizando estudos nos quais cada vez mais homens tenham vez, voz e porque não dizer, mais visibilidade no campo da saúde.

O tema saúde do homem no Brasil teve os seus primeiros debates na década de 1970. Estas discussões se caracterizavam por relacionar o modelo hegemônico de masculinidade a déficits e/ ou agravos à saúde (Gomes \& Nascimento, 2006). No entanto, o avanço dessas discussões deu-se somente após a incorporação das relações de gênero como determinantes do processo de adoecimento e do reconhecimento da saúde como um direito dos homens brasileiros (Brasil, 2008).

Burille, Andréia; Gerhardt, Tatiana Engel (2013). Conexões entre Homens e Saúde: discutindo algumas arranhaduras da masculinidade. Athenea Digital, 13(2), 259-266. Disponible en http://psicologiasocial.uab.es/athenea/index.php/atheneaDigital/article/view/Burille 
De acordo com os princípios do Sistema Único de Saúde, a assistência deve ser equitativa, no entanto, no cotidiano dos serviços de saúde observa-se uma lacuna com relação às ações e práticas que priorizem os homens. Dessa forma, visualiza-se a necessidade emergente de sensibilização do Sistema de Saúde para atender às particularidades da população masculina, para assim Ihes ofertar, de forma eficiente, serviços compatíveis com as suas necessidades.

Ao reconhecer que os agravos do sexo masculino constituem sérios problemas de saúde pública (Brasil, 2008), o Ministério da Saúde, em 2008, juntamente com gestores do Sistema Único de Saúde, com a sociedade científica e civil e com agências de cooperação internacional, lançou a Política Nacional de Atenção Integral à Saúde do Homem (PNAISH), a qual traduz um longo anseio da sociedade.

Buscando colocar em pauta o tema saúde do homem a PNAISH (Brasil, 2008) aponta estudos (Araújo \& Leitão, 2005; Gomes, Nascimento \& Araújo, 2007; Gomes, Nascimento, Rebello \& Araújo, 2008) que evidenciaram algumas justificativas para a ausência de homens nos serviços de saúde, sendo estas alocadas em dois grupos principais de determinantes, aqueles que se estruturam como barreira institucional e os de natureza sociocultural. Ilustrando um pouco mais essas barreiras, têm-se o horário de funcionamento dos serviços que coincidem com o horário de trabalho, o tempo de espera para atendimento e profissionais de saúde, em sua grande maioria pertencente ao sexo feminino, como elementos constituintes da barreira institucional. Já a barreira sociocultural está estruturada no que é prescrito socialmente ao ser homem, ou seja, ser forte, sentir-se invulnerável, viril, entre outras prescrições atribuídas ao sexo masculino.

Neste momento não pretendemos aprofundar a discussão acerca da barreira institucional, embora reconheçamos a importância da mesma na ausência ou baixa frequência dos homens nos serviços de saúde. Focaremos o olhar sobre o modelo de masculinidade reproduzido e difundido pela sociedade (e pela própria política de saúde do homem) e sobre a barreira sociocultural e suas repercussões na saúde. Para isso trazemos como proposta de discussão dois importantes vetores: a construção social da masculinidade e as arranhaduras que podem ser causadas na mesma.

\section{Metodologia}

Este texto traz algumas reflexões oriundas do seminário temático "Ser homem na contemporaneidade: discutindo algumas arranhaduras da masculinidade" apresentado no Seminário Internacional Rotas Críticas III, realizado na cidade de Porto Alegre, Brasil, em 2011, e, por conseguinte, discutir o processo de socialização masculino. Para finalizar buscamos pontuar alguns aspectos da vida cotidiana que podem colocar em risco/ameaçar ou arranhar a masculinidade, como buscar cuidado e até mesmo o próprio processo de envelhecimento.

O seminário no qual abordamos as conexões entre saúde e homens contou com a participação de 17 pessoas, homens e mulheres de diferentes regiões do estado do Rio Grande do Sul, assim como participantes de outros estados brasileiros. Engajados em discutir o tema apresentaram-se jornalistas, publicitários, professores, enfermeiros, assistentes sociais, psicólogos, membros de delegacia da mulher (delegados; delegadas), policial civil, advogado, estudantes da área das ciências sociais, entre outros profissionais. Foi proposta ao grupo a seguinte questão "Ser homem é...". Para isso, ofertamos material gráfico para que os participantes pudessem se expressar na forma de cartazes. $O$ trabalho transcorreu em pequenos grupos e após, os trabalhos foram apresentados para o grande grupo. 


\section{Discussões}

Fazendo uma síntese das apresentações observamos que em muitos cartazes o homem surge acompanhado de uma bela mulher, o homem como garanhão, "pegador", foi ilustrado por personagens de telenovelas brasileiras. Lázaro Ramos na novela global Insensato Coração, foi considerado como o cara do momento, sem falar do ator Wagner Moura colocado como o homem ideal, forte e protetor. Houve espaço para as famosas tríades homem-mulher-cerveja, homem-cerveja-futebol e homemmulher-carro. Tais ilustrações estão fortemente vinculadas a uma masculinidade heteronormativa, a qual se constitui como um modelo hegemônico sobre as demais masculinidades.

Também surgiu a noção do homem como provedor, protagonista de negócios e de grandes obras, forte fisicamente e decidido emocionalmente. Alguns dos participantes selecionaram imagens relacionadas à paternidade e à violência. Um dos grupos trouxe a imagem de Michael Jackson como "ser complexo assexual" aprisionado dentro de um corpo biológico masculino, o que gerou muita discussão. Outro ponto marcante é que um dos grupos apresentou uma figura de um homem com uma mulher e a frase "ao homem é permitido transgredir" o que fez o grande grupo discutir as questões dos papéis sociais referentes as mulheres e os referentes aos homens.

$\mathrm{Na}$ sequência, foram apontados dois elementos interessantes para a discussão que consistiram, por um lado, na necessidade de se pensar o "ser homem" em sua diversidade, embora apontada apenas nos aspectos físicos e étnicos, sem que fosse discutida a sua relação com os modelos de masculinidades; e por outro lado, a expressão de "ser homem no sentido mais profundo" apontado como significado de responsabilidades sociais a serem assumidas na sociedade atual.

Assim, pareceu que para a grande maioria dos participantes "ser homem é..." pertencer a uma masculinidade heteronormativa, a qual configura-se como modelo hegemônico e que se sobressaí sobre os demais modelos. É cumprir com as prescrições que estão enraizadas em nossa sociedade, nem que para isso tenham que colocar em risco sua saúde. Tais concepções apresentadas não surgiram no momento do seminário e sim se encontram alicerçadas em uma sociedade que estrutura papéis e dita prescrições em função do gênero masculino e feminino.

Em relação a esses elementos, Fátima Regina Ceccheto (2004) assinala que as diferenças entre homens e mulheres foram, por muito tempo, consideradas incontestavelmente inscritas na natureza, isto é, no corpo biológico. Os papéis familiares, sociais e profissionais, por séculos permaneceram distintos e condicionantes das práticas de socialização de homens e mulheres. Assim, a construção social do feminino e do masculino foi-se impondo como própria da natureza e imutável (Silva, 1999). No entanto, surge com o feminismo moderno, na década de 1970, a constatação de que as relações de gênero variam de cultura para cultura e de época para época. Assim, a masculinidade deixou de ser algo evidente e, portanto, inquestionada, passando a ser discutida cada vez mais (Cecchetto, 2004).

Ao falar de masculinidades e feminilidades torna-se pertinente trazer o conceito de elaborado por Joan Scott (1995), a qual coloca que gênero é uma construção social sobreposta a um corpo sexuado. É construído e alimentado com base em símbolos, normas e instituições que definem modelos de masculinidade e feminilidade e padrões de comportamento aceitáveis ou não para homens e mulheres. Desse modo, o gênero delimita campos de atuação para cada sexo, dá suporte à elaboração de leis e suas formas de aplicação. Também está incluída no gênero a subjetividade de cada sujeito, sendo única sua forma de reagir ao que lhe é oferecido em sociedade. Neste contexto, Carole S. Vance (1995) afirma que foi a partir do construtivismo social que o gênero pôde ser percebido por meio de significados 
culturais e de relações de poder que o constroem rejeitando formas óbvias de essencialismo e universalização.

A masculinidade numa perspectiva de gênero pode ser vista como um espaço simbólico que serve para estruturar a identidade do ser homem, através de atitudes e comportamentos que devem ser seguidos por aqueles que desejam receber o certificado de masculinidade e não serem questionados e nem vítimas de estigma por parte daqueles que compartilham dessas prescrições (Oliveira, 2004). Dentre os diversos modelos de masculinidade, alguns são mais valorizados e exaltados, enquanto outros são desprezados e subordinados (Cecchetto, 2004).

Como mencionado, as masculinidades são construídas histórica e sócio culturalmente, sendo que sua significação é um processo em constante construção e transformação na sociedade. Entre os distintos modelos de masculinidade, aquele que consegue ser mais apreciado, com maior legitimidade, e se apropriar de outros modelos, reunindo maior poder, pode ser considerado hegemônico. Tal modelo, idealmente estabelecido e dificilmente seguido por todos os homens, consiste numa referência que, ao mesmo tempo, se impõe e se relaciona com os modelos alternativos ou subordinados. No modelo de masculinidade hegemônica, destacam-se como eixos estruturantes a dominação e a heterossexualidade (Cecchetto, 2004; Gomes et al, 2008).

Com relação aos eixos estruturantes da masculinidade hegemônica, enfatiza-se que existe um senso comum que atribui à identidade masculina a imagem de um ser forte, capaz, protetor, decidido e corajoso. Estas atribuições estão enraizadas em muitas culturas, nas quais a educação dos meninos segue padrões de oposição entre os gêneros, uma vez que são orientados para serem provedores e protetores e também aprendem desde cedo a suportar suas dores físicas e emocionais sem chorar (Braz, 2005; Gomes et al, 2007).

Ao mesmo tempo, na socialização dos meninos, a violência também é estimulada pela educação já que são ensinados a revidar em situação de agressão e incentivados a praticar esportes em que a violência está presente e é aceita (Braz, 2005). Desta forma, o padrão de masculinidade é idealizado por meninos e homens não pelo desejo de serem viris, e sim pelo medo de serem vistos como afeminados (Sabo, 2002). Pode-se então dizer que meninos são historicamente criados para aprender a serem homens, ou melhor, parafraseando Simone de Beauvoir, não se nasce homem, torna-se homem.

Essa distinta socialização de meninos e meninas inicia cedo, na própria família, que perpetua as desigualdades entre homens e mulheres (Okin, 2008). Meninas ganham quarto rosa e meninos quarto azul; os presentes também são diferentes, para meninos dão-se carrinhos e bolas e para meninas bonecas e panelinhas para brincar. Desde a infância começa a ser veiculada a asserção que meninas devem cuidar e que meninos, a exemplo do pai, devem atuar fora de casa. Outro fato que também necessita ser refletido pela sociedade é que se uma menina pede para ganhar um carrinho não é recriminada por isso, mas se um menino pede aos pais uma boneca ou gosta de brincar com panelinhas, este é vítima de chacota e sua masculinidade passa a ser questionada.

Assim, a família, a escola e a sociedade em geral vão assujeitando o indivíduo para que ocupe o lugar de homem ou de mulher - que the cabe no espaço social. Dentro desta lógica, estrategicamente, os meios de comunicação de massa, especialmente a televisão, assim como os brinquedos, os jogos eletrônicos, cinema, música, etc., formam um conjunto de procedimentos pedagógicos que vão educando, subliminarmente, meninos e meninas a incorporarem os gêneros masculino e feminino (Santos, 2010). 
Ao falar de gênero e atores masculinos, Daniel Korin (2001) pontua que os homens aprendem a desligarse das qualidades identificadas como femininas. O desejo e a capacidade de cuidar desaparecem durante a socialização, que impõe que o homem necessita apresentar capacidades masculinas como autonomia, força, racionalidade, repressão das emoções, sucesso e poder. Além disso, este autor ressalta que os homens, muitas vezes, se encontram divididos entre os papéis atribuídos pela sociedade e as suas necessidades. Se decidirem realizar seu papel masculino prescrito pelo social, suas necessidades ficam insatisfeitas, resultando em condutas compensadoras, agressivas e de risco, que os predispõem a doença, lesões e morte, e inclusive causam danos às pessoas que os rodeiam. Ao serem satisfeitas as suas necessidades, mas não as expectativas da sociedade, o homem pode terminar sendo considerado pouco homem pelos outros ou, inclusive, por si mesmo.

Complementando Korin (2001), assinala-se que em uma sociedade estruturada pelo gênero como a nossa, o não cumprimento dos papeis sociais atribuídos à figura masculina pode desencadear sentimentos de inferioridade e sensação de não pertencimento ao universo masculino. Mas ao mesmo tempo, manter este ideal de masculinidade que é ensinado desde a infância ao menino é um fator de risco para os homens, em um processo de negação das vulnerabilidades físicas e psíquicas (Braz, 2005). Neste contexto, também cabe destacar a existência de certo "silenciamento" em torno da manutenção desta identidade heróica atribuída aos homens, o que por sua vez acaba dificultando a busca por cuidado e atenção, já que foram criados para assistir e prover.

Nesse cenário, relacionar a construção social da masculinidade e o processo de adoecimento torna-se relevante, uma vez que a manutenção de papéis e de características atribuídas aos homens pode influenciar na escolha de buscar ou não cuidado. Assim, ao adotar a expressão "arranhaduras da masculinidade"1 parte-se do pressuposto que buscar cuidado em saúde aproximaria os homens de um espaço de representações de feminilidade, já que o cuidado normalmente é associado a práticas femininas. Tal fato precisa ser considerado já que repercute nas questões de saúde e pode influenciar nas trajetórias masculinas em busca de cuidado, pois no modelo hegemônico de masculinidade, o qual é considerado heteronormativo, a busca por cuidado pode causar "arranhaduras na masculinidade".

O adoecer é considerado um sinal de fragilidade para os homens que não reconhecem esta situação como um processo inerente à sua própria condição biológica. Além disso, o cultivo do pensamento mágico de que nunca irão ficar doentes faz com que os homens se sintam invulneráveis e consequentemente, se exponham mais vulnerabilizando-se (Braz, 2005; Gomes et al, 2007; Medrado, Lyra-da-Fonseca, Leão, Lima \& Santos, 2005; Sabo, 2002).

Andréia Burille (2009) ao realizar um trabalho com homens em tratamento radioterápico observou que estes não cuidavam da saúde. Alguns dos entrevistados, apesar de terem consciência que a saúde se encontrava debilitada, não buscaram auxílio no momento, deixando para procurar por cuidado somente quando a situação já estava agravada. Este achado concorda com outros estudos, que observaram a entrada tardia dos homens no sistema de saúde, já nos níveis de média e alta complexidade, tendocomo consequência o agravamento da patologia devido o retardo no atendimento (Figueiredo, 2005; Pinheiro, Viacava, Travassos \& Brito, 2002).

Em um estudo realizado nos Estados Unidos, Will Courtenay (2000) observou que os homens estão mais propensos a adoção de crenças e comportamentos que oferecem riscos a saúde, e são menos predispostos a participar de ações que visem à promoção da saúde e a longevidade. Para o autor, as

\footnotetext{
${ }^{1} \mathrm{O}$ termo "Arranhaduras na masculinidade" é de autoria de Romeu Gomes, o qual utiliza este termo para referir-se ao exame de toque retal como arranhador da masculinidade (Gomes et. al, 2008). Neste trabalho, considera-se que a masculinidade é arranhada pela busca de cuidado em geral, seja ele oferecido pelo sistema profissional, popular ou informal.
} 
crenças e os comportamentos representam uma forma que os homens encontram para demonstrar sua masculinidade.

Alguns trabalhos têm revelado que homens e mulheres apresentam comportamentos e representações distintas no que se refere à saúde. Em geral, as mulheres buscam mais os serviços de saúde do que os homens (Laurenti; Mello-Jorge \& Gotlieb, 2005; Silva \& Alves, 2003), tendem a declarar mais as suas doenças, consumirem mais medicamentos e se submetem a mais exames, como também avaliam pior seu estado de saúde (Silva \& Alves, 2003). Destaca-se que as motivações pela busca por cuidado também diferem entre os sexos, as mulheres vão mais aos serviços de saúde com intuito de realizar exames de rotina e ações preventivas, já os homens procuram auxílio por motivo de doença (Pinheiro et al, 2002).

Um estudo realizado por Romeu Gomes, Elaine Ferreira do Nascimento e Fábio Carvalho de Araújo (2007) revelou que os homens reconhecem que procuram menos os serviços de saúde ao serem comparados com as mulheres, sendo que a justificativas mais presentes giraram em torno de um único argumento: os papéis atribuídos à identidade masculina. Para os autores o ser homem estaria associado à invulnerabilidade, força, coragem e virilidade, características incompatíveis com a demonstração de fraqueza e de medo. Nesta concepção, procurar ajuda colocaria em risco à própria masculinidade e aproximaria os homens dos comportamentos femininos.

Marlene Braz (2005) ao colocar os riscos oferecidos pelos papéis de gênero aponta que a mulher é onerada por meio da desvalorização de seu papel social enquanto o homem paga com sua vida. Assim, evidencia-se que as questões de gênero influenciam a conduta e os hábitos de vida masculinos, produzindo não apenas modos de vida, mas também, maneiras de adoecer e morrer. Ao realizar uma pesquisa com homens em situação de adoecimento crônico, Andréia Burille (2011) evidencia que pensar, falar e vivenciar uma doença arranha a masculinidade, portanto, os homens evitam esse assunto.

Outra forma de arranhaduras da masculinidade decorre do processo inerente de envelhecimento humano. Tal fato pode ser comprovado a partir da invenção da andropausa, a menopausa masculina. Assim a partir dos cinquenta anos de idade homens teriam um declínio de suas funções sexuais, sendo necessário intervir e incluir em seu cotidiano hormônios para garantir sua virilidade e desse modo manter sua masculinidade intacta, sem arranhaduras.

Bárbara Marshall (2007) ao falar da andropausa pontua que no final do século XX ocorreu um aumento do interesse científico e comercial na promoção da menopausa masculina como um distúrbio clínico que poderia ser tratado com a substituição de hormônios (testosterona). Seria esta uma tentativa de medicalizá-la e tratá-la. Segundo a autora, o acesso ao Viagra proporcionou novos impulsos à medicalização da sexualidade masculina na meia idade, embora haja convergências entre os interesses científicos e comerciais dessa medicalização masculina. No trabalho Testosterone Dreams (2005) John Hoberman coloca os razões que levaram a Testoterona a adquirir tanta aceitação e status entre os homens. Também coloca que o uso de desse hormônio mexe com as inseguranças masculinas e que seu uso dá-se, muitas vezes de maneira indiscriminada.

Ainda sobre este assunto, Marshall (2007, p. 512) coloca que "a função sexual agora serve como um veículo para a reconstrução da masculinidade como 'Eterna' na medida em que os homens podem demonstrar sua virilidade, podem ainda ser homens e afastar a velhice e a perda de status". Desse modo, a diminuição do desejo sexual e o declínio de hormônios, processos fisiológicos que fazem parte envelhecimento, surgem como uma ameaça aos homens de meia idade, sendo necessário buscar 
intervenção clínica. Compartilhamos da idéia de Gomes (2008), que pontua que a sexualidade saudável é caracterizada por "uma experiência de uma vida sexual prazerosa, informada, sem riscos de doenças, livre de violência e baseada na auto-estima" (p. 24). Assim, uma sexualidade saudável não deve ser avaliada somente pelo desempenho sexual, é preciso ampliar o leque, considerar a sexualidade em sua plenitude.

\section{Considerações Finais}

Para finalizar esse trabalho, reforçamos que os homens precisam se reconhecer e também serem reconhecidos pela sociedade como portadores de direitos a saúde, inclusive de uma sexualidade saudável, compatível como o processo de senilidade. Assim, ao refletir acerca das influencias geradas pelo modelo hegemônico de masculinidade, que prescreve e disciplina os corpos masculinos, pode se observar que este ao tentar corresponder este modelo, os homens muitas vezes se tornam vulneráveis, em especial nas questões que envolvem o viver saudável, e por que não pensar que se colocam em "situações-limite", que aumentam a probabilidade de sofrimento, adoecimento e morte.

\section{Referências}

Araújo, Maria Alix Leite \& Leitão, Glória da Conceição Mesquita (2005). Acesso à consulta a portadores de doenças sexualmente transmissíveis: experiências de homens em uma unidade de saúde de Fortaleza, Ceará, Brasil. Cad. Saúde Pública, 21(2), 396-404.

Braz, Marlene (2005). A construção da subjetividade masculina e seu impacto sobre a saúde do homem: reflexão bioética sobre justiça distributiva. Ciênc. saúde coletiva, 10(1), 97-104.

Brasil (2008). Ministério da Saúde. Departamento de Ações Programáticas e Estratégicas. Política Nacional de Atenção Integral à Saúde do Homem. Brasília: Autor.

Burille, Andréia (2009). Conhecendo as mudanças no cotidiano de homens com câncer: um olhar sobre a perspectiva de gênero. Trabalho de Conclusão de Curso inédito. Universidade Federal de Pelotas, Faculdade de Enfermagem.

Burille, Andréia (2011). Itinerários terapêuticos de homens em situação de adoecimento crônico: a busca por cuidado e as arranhaduras da masculinidade. Dissertação, inédito. Universidade Federal do Rio Grande do Sul, Escola de Enfermagem.

Cecchetto, Fátima Regina (2004). Violência e estilos de masculinidade. Rio de Janeiro: FGV Editora.

Courtenay, Will (2000). Constructions of masculinity and their influence on men' well-eing: a theory of gender and health. Soc. Sci Med, 50(1), 385-401.

Figueiredo, Wagner dos Santos (2005). Assistência à saúde dos homens: um desafio para os serviços de atenção primária. Ciênc. Saúde Coletiva, 10, 105-9.

Gomes, Romeu (2008). Sexualidade Masculina, Gênero e Saúde. Rio de Janeiro: FIOCRUZ.

Gomes, Romeu \& Nascimento, Elaine (2006). A produção do conhecimento da saúde pública sobre a relação homem-saúde: uma revisão bibliográfica. Cad. Saúde Pública, 22(5), 901-911.

Gomes, Romeu; Nascimento, Elaine Ferreira do \& Araújo, Fábio Carvalho de (2007). Por que os homens buscam menos os serviços de saúde do que as mulheres? As explicações de homens com baixa escolaridade e homens com ensino superior. Cad. Saúde Pública, 23(3), 565-74.

Gomes, Romeu; Nascimento, Elaine Ferreira do; Rebello, Lúcia Emilia Figueiredo de Souza \& Araújo, Fábio Carvalho de (2008). As arranhaduras da masculinidade: uma discussão sobre o toque retal como medida de prevenção do câncer prostático. Ciênc. Saúde Coletiva, 13 (6), 19751984. 
Hoberman, John (2005). Testosterone dreams. Rejuvenation, aphrodisia, doping. Berkeley: Univ. of California Press.

Korin, Daniel (2001). Nuevas perspectivas de género em salud. Rev. AdolescLatinoam, 2(2), 67-79.

Laurenti, Ruy; Mello-Jorge, Maria Helena Prado \& Gotlieb, Sábina Léa Davidson (2005). Perfil epidemiológico da morbi-mortalidade masculina. Ciênc. Saúde coletiva, 10, 35-46.

Marhall, Bárbara (2007). Climatericredux?: (Re) medicalizing the male menopause. Men and masculinity, 9, 509-529.

Medrado, Benedito; Lyra-da-Fonseca, Jorge Luís Cardoso; Leão, Luciana Melo de Souza; Lima, Daniel Costa \& Santos, Breno (2005). Homens jovens no contexto do cuidado: leituras a partir da paternidade na adolescência. In Rubens de Camargo Adorno, Augusta Tereza Alvarenga \& Maria da Penha Vasconcelos (Org.), Jovens, trajetória, masculinidades e direitos (pp. 241-64). São Paulo: Fapesp/EDUSP.

Okin, Susan M (2008). Gênero, o público e o privado. Revista Estudos Feministas, 16(2), 305-332. Oliveira, Pedro Paulo (2004). A construção social da masculinidade. Belo Horizonte: Editora UFMG. Pinheiro, Rejane Sobrinho; Viacava, Francisco; Travassos, Claúdia \& Brito, Alexandre dos Santos (2002). Gênero, morbidade, acesso e utilização de serviços de saúde no Brasil. Ciênc. Saúde Coletiva, 7(4), 687-707.

Sabo, Donald (2002). O estudo crítico das masculinidades. In Miriam Adelman \& Celsi Brönstrup Silvestrin (Org.), Coletânea gênero plural (pp. 33-46). Curitiba: Editora UFPR.

Santos, Paulo Reis dos (2010). Desejos, Conflitos e Preconceitos na Constituição de uma Travesti no Mundo da Prostituição. Revista Latino-americana de Geografia e Gênero, 1(1), 39-48.

Scott, Joan (1995). Gênero: uma categoria de análise histórica. Educação e Realidade, 20 (2), 71-99. Silva, Luiza Ferreira (1999). O gênero, determinante cultural de saúde. Rev. Epidem, 13(5), 31-34. Silva, Luiza Ferreira \& Alves, Fátima (2003). A saúde das mulheres em Portugal. Porto: Afrontamento. Vance, Carole S. (1995). A antropologia redescobre a sexualidade: um comentário teórico. Physis, 5(1), 7-32.

\begin{tabular}{l}
\hline Este texto está protegido por una licencia Creative Commons. \\
Usted es libre de copiar, distribuir y comunicar públicamente la obra bajo las siguientes condiciones: \\
Reconocimiento: Debe reconocer y citar al autor original. \\
No comercial. No puede utilizar esta obra para fines comerciales. \\
Sin obras derivadas. No se puede alterar, transformar, o generar una obra derivada a partir de esta obra. \\
Resumen de licencia - Texto completo de la licencia
\end{tabular}

